Www.jmscr.igmpublication.org Impact Factor 5.84

Index Copernicus Value: 83.27

ISSN (e)-2347-176x ISSN (p) 2455-0450

crossref DOI: _https://dx.doi.org/10.18535/jmscr/v5i7.84

\title{
Anatomical Differences Between T2 WI FSE and Proton Density Fat Saturation Sequences in MRI Examination of Knee Joint with Sagittal Slice
}

\author{
Authors \\ Gatot Murti Wibowo $^{1 *}$, Siti Masrochah ${ }^{2}$, Irwan Katili ${ }^{3}$, Atina Izzah Kusumaningrum ${ }^{4}$ \\ ${ }^{1-3}$ Department of Radiological Technology, The Health Polytechnic of Semarang, Indonesia \\ ${ }^{4}$ Department of Radiology, PKU Muhammadiyah Hospital Cepu, Indonesia \\ *Corresponding Author
}

Gatot Murti Wibowo

Email: gatotmurtiw@gmail.com

\begin{abstract}
Background: Anterior Cruciate Ligament (ACL) and Posterior Cruciate Ligament (PCL) must be visible for radiologists when performing Magnetic Resonance Imaging (MRI). One of the sequences used to reveal ACL and PCL is T2WI FSE sequence with Proton Density Fat Saturation. The difference sequence will result in different image quality.

Objective: The aim of the research is to find the difference anatomy information between T2WI FSE sequence and Proton Density Fat Saturation on sagittal slice and to identify what sequence is better to reveal ACL and PCL anatomy.

Methods: This research is experimental queasy. Data consists of 20 images from knee joint MRI sagittal slices from 10 volunteers with two variation procedures: T2WI FSE and Proton Density Fat Saturation. 20 pictures of ACL and PCL anatomy were reviewed by three experienced radiologists in MRI. Data were analyzed with Wilcoxon test.

Results: The result of this research is showing the difference of anatomy information between T2WI FSE sequence with Proton Density Fat Saturation on MRI examination for Knee Joint sagittal slices.The result indicates that the mean rank value of T2WI FSE sequence (4.50) is better than Proton Density Fat Saturation sequence (0.000).

Conclusion: Since there is a different anatomy information between the two sequences on MRI knee joint sagittal slices, the T2WI FSE sequence is recommended for showing anatomy information of ACL and PCL. Keyword: Knee Joint, T2WI FSE, Proton Density Fat Saturation, Anterior Cruciate Ligament (ACL) dan Posterior Cruciate Ligament (PCL).
\end{abstract}

\section{INTRODUCTION}

Anterior Cruciate Ligament (ACL) and Posterior Cruciate Ligament (PCL) are one of the ligaments in the knee. That ligament is white structures functioning as connectors to the other ligaments binding the bone to another and stabilizing the knees (Schmitz, 2012).
Various pathologies happen in the knee joint, one of them is trauma on Anterior Cruciate Ligament (ACL) and Posterior Cruciate Ligament (PCL). According to Allen (2016), the injury of Posterior Cruciate Ligament (PCL) is less common than the injury on Anterior Cruciate Ligament (ACL), but 
PCL is often not recognized. PCL is wider than the surface and stronger than ACL.

According to Westbrook (2008), the protocol to display Anterior Cruciate Ligament (ACL) and Posterior Cruciate Ligament (PCL) requires three slices: sagittal, coronal, and axial. For sagittal slices, sequences used are coherent GRE T2*/ T2WI FSE/ Proton Density/ and Proton Density Fat Saturation/ T2WI Fat Saturation.

Following the work of Khoury et.al (2011) that determines the accuracy of rate internal of knee derangement, the author would like to examine more deeply about the using T2WI FSE sequence, and Proton Density Fat Sat to reveal ACL and PCL.

In most MRI examination cases of the knee joint,sagital slices often used is T2WI FSE or Proton Density Fat Sat.

The aim of this research is to know the difference of anatomy information of T2WI FSE sequence and Proton Density Fat Saturation series on MRI knee joint examination with sagital slices in the study of Anterior Cruciate Ligament (ACL) and Posterior Cruciate Ligament (PCL). Furthermore, te study wants to know which sequence is better to be used on a usual protocol of MRI knee joint to rate Anterior Cruciate Ligament (ACL) and Posterior Cruciate Ligament (PCL).

\section{METHOD}

This research is queasy controlled experimental study design. Ten people are taken as samples undergoing the MRI examination of the knee joint with two different sequences on each: the T2WI FSE and the Proton Density Fat saturation.

Exclusion criteria in this research are the healthy volunteers who have never experienced the trauma on his/her knee joint, not suffering from painful on knee joint area, and have never done the treatment of arthritis or steroid. While the inclusion criteria are those between the ages of $<30$ years, weigh $<60$ $\mathrm{kg}$ or have healthy BMI. The respondents which giving the rate for MRI knee joint image are 3 (three) radiologists.

The procedures of the research are:
1. Volunteers are given an inform consent about purpose and objectives of this research and explained about the examination procedures, and reassured that this research is safe.

2. Methods to perform the MRI image of the knee joint with T2WI FSE and sequence Proton Density Fat saturation parameters are determined.

3. Every volunteer was scanned twice each with T2WI FSE and Proton Density Fat saturation sequence.

4. The image results were saved in CD room with DICOM format without any identifying information, only codes of the serial number for the image.

5. 3 (three) experienced radiologists in MRI give appraisals of MRI knee joint models. The appraisal is done to anatomy information, covering the intensity level of Anterior Cruciate Ligament (ACL) and Posterior Cruciate Ligament (PCL) area, and the limit on the surrounding structures (borderline).

6. The appraisal was done by giving checklist $(\sqrt{ })$ sign on questioners provided. Assessments criteria are 4 points for "Very Clear," 3 points for "Clear," 2 points for "Somewhat Not Clear," and 1 point for "Not Clear."

The Ordinal data with two group of variables were tested with Wilcoxon test to assess the differences mean between the two variables with the significance level of $95 \%$. The highest value of mean rank is used to find out the better sequence in revealing the anatomy information of ACL and PCL.

\section{RESULTS}

The research samples are described in the following table:

Table 1. The Distribution of Sample According to Genders

\begin{tabular}{ccc}
\hline Gender & N (Number) & Percentages (\%) \\
\hline Men & 3 & $30 \%$ \\
Women & 7 & $70 \%$ \\
\hline Total & 10 & $100 \%$ \\
\hline
\end{tabular}


Table 2 The Distribution Sample According to Ages

\begin{tabular}{lll}
\hline Age & N (Number) & $\begin{array}{c}\text { Percentages } \\
(\%)\end{array}$ \\
\hline $19-21$ & 1 & $10 \%$ \\
$22-24$ & 6 & $60 \%$ \\
$25-27$ & 2 & $20 \%$ \\
$28-30$ & 1 & $10 \%$ \\
\hline Total & 10 & $100 \%$ \\
\hline
\end{tabular}

Table 3 The Distribution of Sample According to Weights

\begin{tabular}{lcc}
\hline Weight & N (Number) & Percentages (\%) \\
\hline $50-52$ & 2 & $20 \%$ \\
$53-55$ & 4 & $40 \%$ \\
$56-58$ & 2 & $20 \%$ \\
$59-61$ & 2 & $20 \%$ \\
\hline Total & 10 & $100 \%$ \\
\hline
\end{tabular}

The result of MRI knee joint images using T2WI FSE and Proton Density (PD) Fat Saturation sequence is presented below.
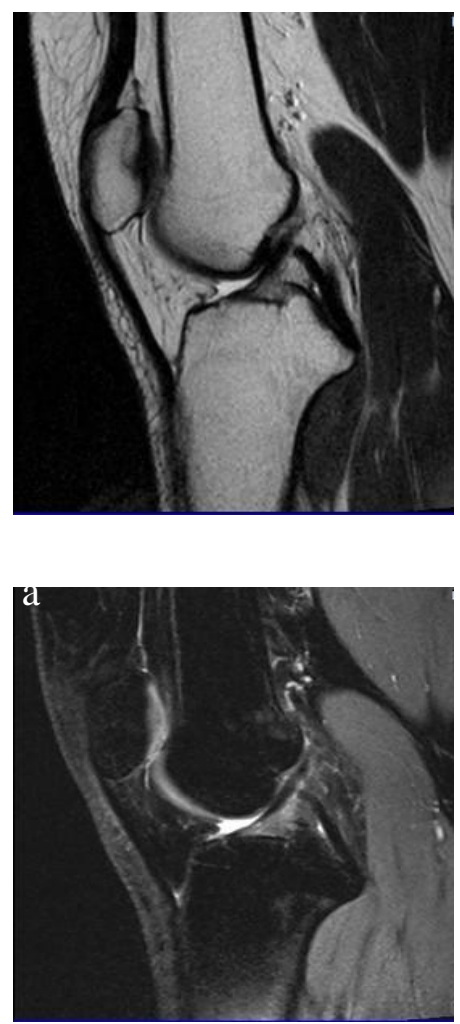

Figure 1. Sagittal Slice of T2WI FSE (a) and Proton Density Fat Saturation (b)

Cohen's Kappa test is performed to identify the level of agreement or objectivity from three radiologists before processing to Wilcoxon Test. The result of Kappa test are depicted below:
Table 4. Kappa Test for T2WI FSE Value and Proton Density Fat Saturation

\begin{tabular}{lccccc}
\hline \multirow{2}{*}{ Sequence } & \multicolumn{3}{c}{ Cohen's Kappa' Levels } & & \\
\cline { 2 - 4 } & $\mathrm{R} 1 * \mathrm{R} 2$ & $\mathrm{R} 2 * \mathrm{R}$ & $\mathrm{R} 2 * \mathrm{R}$ & Mean & Note \\
& & 3 & 3 & & \\
\hline T2WI FSE & 0.696 & 0.650 & 0.550 & 0.632 & Good \\
$\begin{array}{l}\text { Proton } \\
\text { Density FS }\end{array}$ & 0.705 & 0.578 & 0.554 & 0.612 & Good \\
\hline
\end{tabular}

The highest value levels found on respondent 1 and respondent 2 are on T2WI FSE sequence (0.696) and proton density Fat Saturation sequence (0.705). According to Altman (1991), the coefficient value of Kappa between 0.61 until 0.80 shows a good agreement value levels, so the respondent 1 and respondent two are considered having the same perspective in appraising.

The result of Wilcoxon Test on the comparison of anatomy information between T2WI FSE and Proton Density Fat Saturation Sequences indicates that the $p$ value $=0.05$. This value implies the acceptance of $\mathrm{H}_{1}$ that there is a significant difference in anatomy information between T2WI FSE and Proton Density Fat Saturation sequences when performing the knee joint MRI examination sagittal slice.

The result of Wilcoxon test for anatomy criteria are:

Table 5. The Result of Wilcoxon Test per Anatomy Criteria Between T2WI FSE and Proton Density Fat Saturation sequences

\begin{tabular}{ll}
\hline Image Information & p-value \\
\hline $\begin{array}{l}\text { Intensity of ACL (T2WI FSE) } \\
\text { Intensity of ACL (Proton Density Fat Saturation) }\end{array}$ & 0.046 \\
$\begin{array}{l}\text { Border Line of ACL ( T2WI FSE) } \\
\text { Borderline of ACL ( Proton Density Fat Saturation) } \\
\text { Intensity of PCL (T2WI FSE) }\end{array}$ & 0.034 \\
$\begin{array}{l}\text { Intensity of PCL ( Proton Density Fat Saturation) } \\
\text { Borderline of PCL ( T2WI FSE) }\end{array}$ & 0.035 \\
$\begin{array}{l}\text { Borderline of PCL ( Proton Density Fat Saturation) } \\
\quad\end{array}$ & 0.034 \\
\hline
\end{tabular}

Since all the p-value is below 0.05 , it is concluded that there is a different information on anatomy criteria of ACL intensity, the borderline of ACL, intensity of PCL, and borderline of PCL. To determine the better anatomy criteria mean rank result was presented in the following table: 
Table 6. The Result of Statistic Wilcoxon Test of Mean Rank on Anatomy Information between T2WI FSE and Proton Density Fat Saturation sequence

\begin{tabular}{lcc}
\hline Criteria & Sequence & Mean rank \\
\hline Intensity of ACL & $\begin{array}{l}\text { Proton Density } \\
\text { Fat Saturation } \\
\text { T2WI FSE }\end{array}$ & 2.50 \\
& $\begin{array}{l}\text { Proton Density } \\
\text { Fat Saturation } \\
\text { T2WI FSE }\end{array}$ & 0.25 \\
\hline Bntensity of PCL & $\begin{array}{l}\text { Proton Density } \\
\text { Fat Saturation } \\
\text { T2WI FSE }\end{array}$ & 3.00 \\
\hline Border Line of PCL & $\begin{array}{c}\text { Proton Density } \\
\text { Fat Saturation }\end{array}$ & 4.00 \\
& T2WI FSE & 4.57 \\
\hline
\end{tabular}

The result of mean rank Wilcoxon test on table 6 shows that T2WI FSE sequence has a higher value on all of the anatomy criteria, except for borderline of PCL anatomy. Anatomy standards of the borderline between T2WI FSE sequence and Proton Density Fat Saturation sequence have the same mean rank value, that is 4.50 . For the highest mean rank value on T2WI FSE sequence is in intensity anatomy information of PCL that is 4.57 and the highest mean rank value on Proton Density Fat Saturation sequence, it is in the anatomy of borderline PCL, that is 4.50 .

\section{DISCUSSION}

The difference anatomy information between T2WI FSE sequence and Proton Density Fat Saturation sequence on MRI examination of Knee Joint

There is a significant difference in anatomy information between T2WI FSE sequence and Proton Density Fat Saturation on MRI examination of knee joint sagittal slices. However, the result of Wilcoxon test on images information per anatomy criteria some organs were not different significantly, that is on anatomy intensity of ACL. This difference is due to each sequence has its function as well as advantages and disadvantages. The parameters used in T2WI FSE sequences are long TR (> $2000 \mathrm{~ms}$ ) and long TE (> $60 \mathrm{~ms}$ ). The long TR will provide a chance for water to break down and a long TE will be able to detect the signal difference between water and fat. Thus by using T2WI FSE ACL and PCL sequences the hypo-intense and fluid will be visible clearer. This opaque visibility is because of the ACL and PCL structures consist of mostly dense tendon fibers so that the FSE T2WI sequence will appear hypointense. In Proton Density, parameters affecting the image quality is the value of TR and TE. Because Fat Saturation technique is added, the adipose tissue will appear dark, the fat looks dark, the liquid will look bright, the muscle will look dark, and bone will look dark. Also, the Fat Saturation technique is not sensitive to magnetic inhomogeneity so that if the organ structure is not homogeneous, then the organ will not well suppressed making the anatomical picture of ACL and PCL is not so clearly visible.

The better anatomy information value between using T2WI FSE and Proton Density Fat Saturation Observed from the mean rank result for all of the anatomy on Wilcoxon test, T2WI FSE sequence is better in showing anatomy information than Proton Density Fat Saturation sequence. However, anatomy information of images on T2WI FSE and Proton Density Fat Saturation sequence were not all differences. Anatomy information of borderline between T2WI FSE and Proton Density Fat Saturation sequence in fact has the same mean rank value.

T2WI FSE sequence is better that ACL and PCL sequences because there will be an appearance of an increasing signal enhancement if there are some pathologies. A significant increase in F2 T2WI signals is required for example after ligament reconstruction where the patient will be needed for control, and the ACL graft should appear as a tight structure, and the thing to do is needing a signal boost using the FSE T2WI sequence on the sagittal piece.

Meanwhile, according to Berquist (2013), acute tears on T2 weighting or water sensitive sequences will reveal high signal intensity is making easy detection if there are abnormalities in the ligaments. According to George (2014), the use of PD Fat Saturation sequences is useful for identifying pathology with adipose tissue content that will appear dark in PD Fat Saturation. Because Fat Saturation technique is added, pathology process will usually look bright on PD Fat Saturation. 
According to Lal, et.al (2000), the sequence of PD Fat Saturation is susceptible to detect bone contusions than FSE PD and T2 weighting.

\section{CONCLUSION}

According to our discussion, some cases can be concluded:

1. There are significant differences of overall image information between T2WI FSE and Proton Density Fat Saturation sequence

2. Mean rank value on T2WI FSE sequence is higher than Proton Density Fat Saturation sequence on all of the images information, except anatomy of borderline PCL. Anatomy of borderline PCL has the same mean rank value that is 4,50 while highest mean rank value on T2WI FSE sequence PCL intensity of anatomy, that is 4,57 and the highest value of mean rank on Proton Density Fat Saturation sequence in borderline PCL anatomy, that is 4,50. In generally, T2WI FSE sequence has better ability than Proton Density Fat Saturation in showing image information. However, Proton Density Fat Saturation sequence should still be used in MRI examination of knee joint because it is good in showing some pathologies such as an acute lesion, bone contusions, and meniscal tears.

\section{BIBLIOGRAPHY}

1. Altman D. G. (1991). Practical statistics for medical research. Chapman \& Hall, London.

2. Berquist, Thomas H, 2013. MRI OF THE Musculoskeletal System Sixth Edition. Wolters Kluwer Lippincott Williams \& Wilkins: Philadelphia.

3. George R, J. Dela Cruz, R. Singh, and Fran Han. 2014. MRI Of Knee. www.mrimaster.com.Accessed on 17 March 2016.

4. Lal, N. R., Jamadar, D. A., Doi, K., Newman, J. S., Adler, R. S., Uri, D. S., \&
Kazerooni, E. A. (2000). Evaluation of bone contusions with fat-saturated fast spin-echo proton-density magnetic resonance imaging. Canadian Association of Radiologists Journal, 51(3), 182.

5. Khoury, N. J., Mahfoud, Z., Masrouha, K. Z., Elkattah, R., Assaad, T., Abdallah, A., \& Hourani, M. H. (2011). The value of Sagittal Fat-Suppressed Proton-Density Fast-SpinEcho of the Knee Joint as a Limited Protocol in Evaluating Internal Knee Derangements. Journal of computer assisted tomography,35(5), 653-661.

6. Schmitz, A. (2012).The importance of including knee joint laxity in dynamic musculoskeletal simulations of movement (Doctoral dissertation, The University of Wisconsin-Madison).

7. Westbrook, Catherine. 2008. Handbook of MRI Technique. Blackwell Science Ltd: United Kingdom. 